\title{
Differential Subordination Results for Fractional Integral Associated with Generalized Mittag-Leffler Function
}

\author{
Abbas Kareem Wanas ${ }^{1}$ and Alaa Hussein Khaleel ${ }^{2}$ \\ Department of Mathematics, College of Science, University of Al-Qadisiyah, Iraq \\ e-mail: abbas.kareem.w@qu.edu.iq ${ }^{1}$; alaa.kahlil@qu.edu.iq ${ }^{2}$
}

\begin{abstract}
In this work, by making use of fractional integral, we define a certain class of holomorphic functions defined by generalized Mittag-Leffler function in the open unit disk $U$. Also, we establish some results for this class related to integral representation, inclusion relationship and argument estimate.
\end{abstract}

\section{Introduction}

Let $\mathcal{A}$ indicate the family of all functions $f$ of the form:

$$
f(z)=z+\sum_{n=2}^{\infty} a_{n} z^{n},
$$

which are holomorphic in the open unit disk $U=\{z \in \mathbb{C}:|z|<1\}$.

For functions $f$ given by (1.1) and $g \in \mathcal{A}$ given by

$$
g(z)=z+\sum_{n=2}^{\infty} b_{n} z^{n},
$$

Received: January 22, 2019; Accepted: February 11, 2019

2010 Mathematics Subject Classification: 30C45.

Keywords and phrases: holomorphic functions, generalized Mittag-Leffler function, fractional integral, subordination, integral representation.

Copyright (C) 2019 Abbas Kareem Wanas and Alaa Hussein Khaleel. This is an open access article distributed under the Creative Commons Attribution License, which permits unrestricted use, distribution, and reproduction in any medium, provided the original work is properly cited. 
the Hadamard product $f * g$ of $f$ and $g$ is defined by

$$
(f * g)(z)=z+\sum_{k=2}^{\infty} a_{n} b_{n} z^{n}=(g * f)(z) .
$$

Given two functions $f$ and $g$ which are analytic in $U$, we say that $f$ is subordinate to $g$, written $f \prec g$ or $f(z) \prec g(z)(z \in U)$, if there exists a Schwarz function $w$ which is analytic in $U$ with $w(0)=0$ and $|w(z)|<1$ such that $f(z)=g(w(z)),(z \in U)$. In particular, if the function $g$ is univalent in $U$, then $f \prec g$ if and only if $f(0)=g(0)$ and $f(U) \subset g(U)$.

The Mittag-Leffler function $E_{\alpha}(z),(z \in \mathbb{C})$ (see [7,8]) is defined by

$$
E_{\alpha}(z)=\sum_{n=0}^{\infty} \frac{z^{n}}{\Gamma(\alpha n+1)}, \quad(\alpha \in \mathbb{C}, \operatorname{Re}(\alpha)>0)
$$

Several researchers have investigated properties of Mittag-Leffler function and generalized Mittag-Leffler function, see for example [4, 5, 9, 10]. Moreover, Srivastava and Tomovski [13] introduced the function $E_{\alpha, \beta}^{\gamma, k}(z),(z \in \mathbb{C})$ in the form:

$$
E_{\alpha, \beta}^{\gamma, k}(z)=\sum_{n=0}^{\infty} \frac{(\gamma)_{n k} z^{n}}{\Gamma(\alpha n+\beta) n !},
$$

where $\alpha, \beta, \gamma \in \mathbb{C}, \operatorname{Re}(\alpha)>\max \{0, \operatorname{Re}(k)-1\}, \operatorname{Re}(k)>0$ and $(x)_{n}$ is the Pochhammer symbol defined by

$$
(x)_{n}=\frac{\Gamma(x+n)}{\Gamma(x)}= \begin{cases}1 & (n=0) \\ x(x+1) \cdots(x+n-1) & (n \in \mathbb{N})\end{cases}
$$

Definition 1.1 [1]. For $f \in \mathcal{A}$ the operator $\mathcal{H}_{\alpha, \beta}^{\gamma, k}: \mathcal{A} \rightarrow \mathcal{A}$ is defined by

$$
\mathcal{H}_{\alpha, \beta}^{\gamma, k} f(z)=Q_{\alpha, \beta}^{\gamma, k}(z) * f(z) \quad(z \in U)
$$

where

$$
Q_{\alpha, \beta}^{\gamma, k}(z)=\frac{\Gamma(\alpha+\beta)}{(\gamma)_{k}}\left(E_{\alpha, \beta}^{\gamma, k}(z)-\frac{1}{\Gamma(\beta)}\right),
$$




$$
\beta, \gamma \in \mathbb{C}, \operatorname{Re}(\alpha)>\max \{0, \operatorname{Re}(k)-1\}, \operatorname{Re}(k)>0 .
$$

By some easy calculations, we have

$$
\mathcal{H}_{\alpha, \beta}^{\gamma, k} f(z)=z+\sum_{n=2}^{\infty} \frac{\Gamma(\gamma+n k) \Gamma(\alpha+\beta)}{\Gamma(\gamma+k) \Gamma(\beta+\alpha n) n !} a_{n} z^{n}
$$

Definition 1.2 [12]. The fractional integral of order $\lambda,(\lambda>0)$ is defined for a function $f$ by

$$
D_{z}^{-\lambda} f(z)=\frac{1}{\Gamma(\lambda)} \int_{0}^{z} \frac{f(\zeta)}{(z-\zeta)^{1-\lambda}} d \zeta
$$

where $f$ is analytic function in a simply-connected region of the $z$-plane containing the origin and the multiplicity of $(z-\zeta)^{\lambda-1}$ is removed by requiring $\log (z-\zeta)$ to be real, when $(z-\zeta)>0$.

We now, by making use of Definition 1.1 and Definition 1.2, we have

$$
D_{z}^{-\lambda} \mathcal{H}_{\alpha, \beta}^{\gamma, k} f(z)=\frac{1}{\Gamma(2+\lambda)} z^{1+\lambda}+\sum_{n=2}^{\infty} \frac{\Gamma(\gamma+n k) \Gamma(\alpha+\beta)}{\Gamma(n+1+\lambda) \Gamma(\gamma+k) \Gamma(\beta+\alpha n)} a_{n} z^{n+\lambda}
$$

It is easily verified from (1.2) that

$$
\begin{aligned}
z\left(D_{z}^{-\lambda} \mathcal{H}_{\alpha, \beta}^{\gamma, k} f(z)\right)^{\prime}= & \left(\frac{\gamma+k}{k}\right) D_{z}^{-\lambda} \mathcal{H}_{\alpha, \beta}^{\gamma+1, k} f(z) \\
& -\left(\frac{\gamma-\lambda k}{k}\right) D_{z}^{-\lambda} \mathcal{H}_{\alpha, \beta}^{\gamma, k} f(z), \quad \operatorname{Re}(\gamma-\lambda k) \neq 0 .
\end{aligned}
$$

Let $T$ be the class of functions $h$ of the form:

$$
h(z)=1+\sum_{n=1}^{\infty} h_{n} z^{n}
$$

which are analytic and convex univalent in $U$ and satisfy the condition:

$$
\operatorname{Re}\{h(z)\}>0, \quad(z \in U) .
$$

We will require the following lemmas in proving our main results. 
Lemma 1.1 [3]. Let $u, v \in \mathbb{C}$ and suppose that $\psi$ is convex and univalent in $U$ with $\psi(0)=1$ and $\operatorname{Re}\{u \psi(z)+v\}>0,(z \in U)$. If $q$ is analytic in $U$ with $q(0)=1$, then the subordination

$$
q(z)+\frac{z q^{\prime}(z)}{u q(z)+v} \prec \psi(z)
$$

implies that $q(z) \prec \psi(z)$.

Lemma 1.2 [6]. Let $h$ be convex univalent in $U$ and $\mathcal{T}$ be analytic in $U$ with $\operatorname{Re}(\mathcal{T}(z)) \geq 0,(z \in U)$. If $q$ is analytic in $U$ and $q(0)=h(0)$, then the subordination

$$
q(z)+\mathcal{T}(z) z q^{\prime}(z) \prec h(z)
$$

implies that $q(z) \prec h(z)$.

Lemma 1.3 [2]. Let $q$ be analytic in $U$ with $q(0)=1$ and $q(z) \neq 0$ for all $z \in U$. If there exists two points $z_{1}, z_{2} \in U$ such that

$$
-\frac{\pi}{2} b_{1}=\arg \left(q\left(z_{1}\right)\right)<\arg (q(z))<\arg \left(q\left(z_{2}\right)\right)=\frac{\pi}{2} b_{2},
$$

for some $b_{1}$ and $b_{2}\left(b_{1}>0, b_{2}>0\right)$ and for all $z\left(|z|<\left|z_{1}\right|=\left|z_{2}\right|\right)$, then

$$
\frac{z_{1} q^{\prime}\left(z_{1}\right)}{q\left(z_{1}\right)}=-i\left(\frac{b_{1}+b_{2}}{2}\right) m \quad \text { and } \quad \frac{z_{2} q^{\prime}\left(z_{2}\right)}{q\left(z_{2}\right)}=i\left(\frac{b_{1}+b_{2}}{2}\right) m \text {, }
$$

where

$$
m \geq \frac{1-|\varepsilon|}{1+|\varepsilon|} \quad \text { and } \quad \varepsilon=i \tan \frac{\pi}{4}\left(\frac{b_{2}-b_{1}}{b_{1}+b_{2}}\right)
$$

\section{Main Results}

We begin this section by defining the function class $\mathcal{M}(\lambda, \gamma, k, \mu, \alpha, \beta, \delta ; h)$ as follows:

Definition 2.1. A function $f \in \mathcal{A}$ is said to be in the class $\mathcal{M}(\lambda, \gamma, k, \mu$, $\alpha, \beta, \delta ; h)$ if it satisfies the following differential subordination condition: 


$$
\frac{1}{1-\delta}\left(\frac{z\left(D_{z}^{-\lambda} \mathcal{H}_{\alpha, \beta}^{\gamma, k} f(z)\right)^{\prime}}{D_{z}^{-\lambda} \mathcal{H}_{\alpha, \beta}^{\gamma, k} f(z)}-\delta\right) \prec h(z)
$$

where $\alpha, \beta, \gamma \in \mathbb{C}, \quad \operatorname{Re}(\alpha)>\max \{0, \operatorname{Re}(k)-1\}, \quad \operatorname{Re}(k)>0, \quad \lambda>0, \quad 0 \leq \delta<p$ and $h \in T$.

In the first theorem, we find integral representation of the class $\mathcal{M}(\lambda, \gamma, k, \mu$, $\alpha, \beta, \delta ; h)$.

Theorem 2.1. Let $f \in \mathcal{M}(\lambda, \gamma, k, \mu, \alpha, \beta, \delta ; h)$. Then

$$
D_{z}^{-\lambda} \mathcal{H}_{\alpha, \beta}^{\gamma, k} f(z)=z \cdot \exp \left[(1-\delta) \int_{0}^{z} \frac{h(w(s))-1}{s} d s\right] \text {, }
$$

where $w$ is analytic in $U$ with $w(0)=0$ and $|w(z)|<1,(z \in U)$.

Proof. Assume that $f \in \mathcal{M}(\lambda, \gamma, k, \mu, \alpha, \beta, \delta ; h)$. It is easy to see that subordination condition (2.1) can be written as follows

$$
\frac{z\left(D_{z}^{-\lambda} \mathcal{H}_{\alpha, \beta}^{\gamma, k} f(z)\right)^{\prime}}{D_{z}^{-\lambda} \mathcal{H}_{\alpha, \beta}^{\gamma, k} f(z)}=(1-\delta) h(w(z))+\delta,
$$

where $w$ is analytic in $U$ with $w(0)=0$ and $|w(z)|<1,(z \in U)$.

From (2.2), we find that

$$
\frac{\left(D_{z}^{-\lambda} \mathcal{H}_{\alpha, \beta}^{\gamma, k} f(z)\right)^{\prime}}{D_{z}^{-\lambda} \mathcal{H}_{\alpha, \beta}^{\gamma, k} f(z)}-\frac{1}{z}=(1-\delta) \frac{h(w(z))-1}{z} .
$$

After integrating both sides of (2.3), we have

$$
\log \left(\frac{D_{z}^{-\lambda} \mathcal{H}_{\alpha, \beta}^{\gamma, k} f(z)}{z}\right)=(1-\delta) \int_{0}^{z} \frac{h(w(s))-1}{s} d s
$$

Therefore, from (2.4), we obtain the required result. 
Next, we establish the following inclusion relationship for the class $\mathcal{M}(\lambda, \gamma, k, \mu, \alpha, \beta, \delta ; h)$.

Theorem 2.2. Let $\operatorname{Re}\left\{(1-\delta) h(z)+\delta+\frac{\gamma-\lambda k}{k}\right\}>0$. Then

$$
\mathcal{M}(\lambda, \gamma+1, k, \mu, \alpha, \beta, \delta ; h) \subset \mathcal{M}(\lambda, \gamma, k, \mu, \alpha, \beta, \delta ; h) .
$$

Proof. Let $f \in \mathcal{M}(\lambda, \gamma+1, k, \mu, \alpha, \beta, \delta ; h)$ and put

$$
q(z)=\frac{1}{1-\delta}\left(\frac{z\left(D_{z}^{-\lambda} \mathcal{H}_{\alpha, \beta}^{\gamma, k} f(z)\right)^{\prime}}{D_{z}^{-\lambda} \mathcal{H}_{\alpha, \beta}^{\gamma, k} f(z)}-\delta\right)
$$

Then $q$ is analytic in $U$ with $q(0)=1$. According to (2.5) and using the relation (1.3), we obtain

$$
\frac{\gamma+k}{k} \frac{D_{z}^{-\lambda} \mathcal{H}_{\alpha, \beta}^{\gamma+1, k} f(z)}{D_{z}^{-\lambda} \mathcal{H}_{\alpha, \beta}^{\gamma, k} f(z)}=(1-\delta) q(z)+\delta+\frac{\gamma-\lambda k}{k}
$$

By logarithmically differentiating both sides of (2.6) with respect to $z$ and multiplying by $z$, we get

$$
q(z)+\frac{z q^{\prime}(z)}{(1-\delta) q(z)+\delta+\frac{\gamma-\lambda k}{k}}=\frac{1}{1-\delta}\left(\frac{z\left(D_{z}^{-\lambda} \mathcal{H}_{\alpha, \beta}^{\gamma+1, k} f(z)\right)^{\prime}}{D_{z}^{-\lambda} \mathcal{H}_{\alpha, \beta}^{\gamma+1, k} f(z)}-\delta\right) \prec h(z) .
$$

Since $\operatorname{Re}\left\{(1-\delta) h(z)+\delta+\frac{\gamma-\lambda k}{k}\right\}>0$, then applying Lemma 1.1 to the subordination (2.7), yields $q(z) \prec h(z)$, which implies $f \in \mathcal{M}(\lambda, \gamma, k, \mu, \alpha, \beta, \delta ; h)$.

Theorem 2.3. Let $f \in \mathcal{A}, 0<a_{1}, a_{2} \leq 1$ and $0 \leq \delta<1$. If

$$
-\frac{\pi}{2} a_{1}<\arg \left(\frac{z\left(D_{z}^{-\lambda} \mathcal{H}_{\alpha, \beta}^{\gamma+1, k} f(z)\right)^{\prime}}{D_{z}^{-\lambda} \mathcal{H}_{\alpha, \beta}^{\gamma+1, k} g(z)}-\delta\right)<\frac{\pi}{2} a_{2}
$$


for some $g \in \mathcal{M}\left(\lambda, \gamma+1, k, \mu, \alpha, \beta, \delta ; \frac{1+A Z}{1+B z}\right),(-1 \leq B<A \leq 1)$, then

$$
-\frac{\pi}{2} b_{1}<\arg \left(\frac{z\left(D_{z}^{-\lambda} \mathcal{H}_{\alpha, \beta}^{\gamma, k} f(z)\right)^{\prime}}{D_{z}^{-\lambda} \mathcal{H}_{\alpha, \beta}^{\gamma, k} g(z)}-\delta\right)<\frac{\pi}{2} b_{2}
$$

where $b_{1}$ and $b_{2}\left(0<b_{1}, b_{2} \leq 1\right)$ are the solutions of the equations:

$$
a_{1}=\left\{\begin{array}{c}
b_{1}+\frac{2}{\pi} \tan ^{-1}\left(\frac{(1-|\varepsilon|)\left(b_{1}+b_{2}\right) \cos \frac{\pi}{2} t}{2(1+|\varepsilon|)\left(\frac{(1+A)(1-\delta)}{1+B}+\delta+\frac{\gamma-\lambda k}{k}\right)}\right. \\
+(1-|\varepsilon|)\left(b_{1}+b_{2}\right) \sin \frac{\pi}{2} t
\end{array}\right), \quad B \neq-1
$$

and

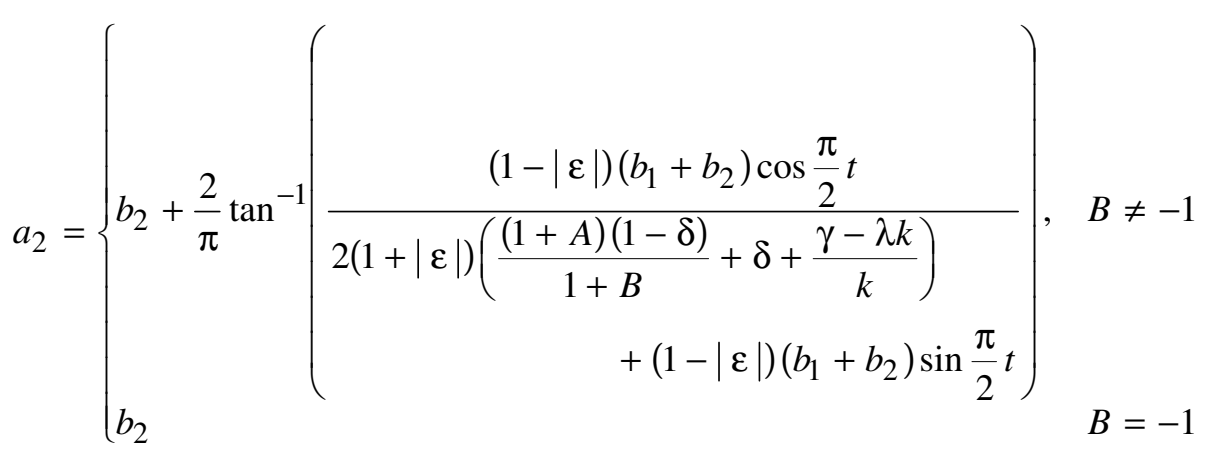

with

$$
\varepsilon=i \tan \frac{\pi}{2}\left(\frac{b_{2}-b_{1}}{b_{1}+b_{2}}\right) \text { and } t=\frac{2}{\pi} \sin ^{-1}\left(\frac{(A-B)(1-\delta)}{\left(\delta+\frac{\gamma-\lambda k}{k}\right)\left(1-B^{2}\right)+(1-\delta)(1-A B)}\right) \text {. }
$$

Proof. Define the function $G$ by 


$$
G(z)=\frac{1}{1-\tau}\left(\frac{z\left(D_{z}^{-\lambda} \mathcal{H}_{\alpha, \beta}^{\gamma, k} f(z)\right)^{\prime}}{D_{z}^{-\lambda} \mathcal{H}_{\alpha, \beta}^{\gamma, k} g(z)}-\tau\right)
$$

where $g \in \mathcal{M}\left(\lambda, \gamma+1, k, \mu, \alpha, \beta, \delta ; \frac{1+A Z}{1+B z}\right),(-1 \leq B<A \leq 1)$, and $0 \leq \tau<1$.

Then $G$ is analytic in $U$ with $G(0)=1$. Therefore by making use of (1.3) and (2.11), we obtain

$$
((1-\tau) G(z)+\tau) D_{z}^{-\lambda} \mathcal{H}_{\alpha, \beta}^{\gamma, k} g(z)=\frac{\gamma+k}{k} D_{z}^{-\lambda} \mathcal{H}_{\alpha, \beta}^{\gamma+1, k} f(z)-\frac{\gamma-\lambda k}{k} D_{z}^{-\lambda} \mathcal{H}_{\alpha, \beta}^{\gamma, k} f(z) .
$$

Differentiating above relation with respect to $z$ and multiplying by $z$, we get

$$
\begin{aligned}
& ((1-\tau) G(z)+\tau) z\left(D_{z}^{-\lambda} \mathcal{H}_{\alpha, \beta}^{\gamma, k} g(z)\right)^{\prime}+(1-\tau) z G^{\prime}(z) D_{z}^{-\lambda} \mathcal{H}_{\alpha, \beta}^{\gamma, k} g(z) \\
= & \frac{\gamma+k}{k} z\left(D_{z}^{-\lambda} \mathcal{H}_{\alpha, \beta}^{\gamma+1, k} f(z)\right)^{\prime}-\frac{\gamma-\lambda k}{k} z\left(D_{z}^{-\lambda} \mathcal{H}_{\alpha, \beta}^{\gamma, k} f(z)\right)^{\prime} .
\end{aligned}
$$

Suppose that

$$
H(z)=\frac{1}{1-\delta}\left(\frac{z\left(D_{z}^{-\lambda} \mathcal{H}_{\alpha, \beta}^{\gamma, k} g(z)\right)^{\prime}}{D_{z}^{-\lambda} \mathcal{H}_{\alpha, \beta}^{\gamma, k} g(z)}-\delta\right)
$$

Using (1.3) again, we have

$$
\frac{\gamma+k}{k} \frac{D_{z}^{-\lambda} \mathcal{H}_{\alpha, \beta}^{\gamma+1, k} g(z)}{D_{z}^{-\lambda} \mathcal{H}_{\alpha, \beta}^{\gamma, k} g(z)}=(1-\delta) H(z)+\delta+\frac{\gamma-\lambda k}{k}
$$

From (2.12) and (2.13), we easily get

$$
G(z)+\frac{z G^{\prime}(z)}{(1-\delta) H(z)+\delta+\frac{\gamma-\lambda k}{k}}=\frac{1}{1-\tau}\left(\frac{z\left(D_{z}^{-\lambda} \mathcal{H}_{\alpha, \beta}^{\gamma+1, k} f(z)\right)^{\prime}}{D_{z}^{-\lambda} \mathcal{H}_{\alpha, \beta}^{\gamma+1, k} g(z)}-\tau\right)
$$


Notice that from Theorem 2.2, $g \in \mathcal{M}\left(\lambda, \gamma+1, k, \mu, \alpha, \beta, \delta ; \frac{1+A Z}{1+B z}\right)$ implies $g \in \mathcal{M}\left(\lambda, \gamma, k, \mu, \alpha, \beta, \delta ; \frac{1+A Z}{1+B z}\right)$. Thus,

$$
H(z) \prec \frac{1+A Z}{1+B z} \quad(-1 \leq B<A \leq 1) .
$$

By using the result of Silverman and Silvia [11], we have

$$
\left|H(z)-\frac{1-A B}{1-B^{2}}\right|<\frac{A-B}{1-B^{2}} \quad(B \neq-1, z \in U)
$$

and

$$
\operatorname{Re}\{H(z)\}>\frac{1-A}{2} \quad(B=-1, z \in U) .
$$

It follows from (2.15) and (2.16) that

$$
\begin{aligned}
& \left|(1-\delta) H(z)+\delta+\frac{\gamma-\lambda k}{k}-\frac{\left(\delta+\frac{\gamma-\lambda k}{k}\right)\left(1-B^{2}\right)+(1-\delta)(1-A B)}{1-B^{2}}\right| \\
& <\frac{(A-B)(1-\delta)}{1-B^{2}}, \quad(B \neq-1, z \in U)
\end{aligned}
$$

and

$$
\operatorname{Re}\left\{(1-\delta) H(z)+\delta+\frac{\gamma-\lambda k}{k}\right\}>\frac{(1-A)(1-\delta)}{2}+\delta+\frac{\gamma-\lambda k}{k}, \quad(B=-1, z \in U) .
$$

Putting

$$
(1-\delta) H(z)+\delta+\frac{\gamma-\lambda k}{k}=\rho e^{i \frac{\pi}{2} \phi},
$$

where

$$
-\frac{(A-B)(1-\delta)}{\left(\delta+\frac{\gamma-\lambda k}{k}\right)\left(1-B^{2}\right)+(1-\delta)(1-A B)}
$$




$$
<\phi<\frac{(A-B)(1-\delta)}{\left(\delta+\frac{\gamma-\lambda k}{k}\right)\left(1-B^{2}\right)+(1-\delta)(1-A B)},(B \neq-1)
$$

and $-1<\phi<1,(B=-1)$, then

$$
\frac{(1-A)(1-\delta)}{1-B}+\delta+\frac{\gamma-\lambda k}{k}<\rho<\frac{(1+A)(1-\delta)}{1+B}+\delta+\frac{\gamma-\lambda k}{k},(B \neq-1)
$$

and

$$
\frac{(1-A)(1-\delta)}{1-B}+\delta+\frac{\gamma-\lambda k}{k}<\rho<\infty,(B=-1) .
$$

An application of Lemma 1.2 with $\mathcal{T}(z)=\frac{1}{(1-\delta) H(z)+\delta+\frac{\gamma-\lambda k}{k}}$, yields $G(z) \prec h(z)$

If there exist two points $z_{1}, z_{2} \in U$ such that

$$
-\frac{\pi}{2} b_{1}=\arg \left(G\left(z_{1}\right)\right)<\arg (G(z))<\arg \left(G\left(z_{2}\right)\right)=\frac{\pi}{2} b_{2},
$$

then by Lemma 1.3, we get

$$
\frac{z_{1} G^{\prime}\left(z_{1}\right)}{G\left(z_{1}\right)}=-\frac{m i}{2}\left(b_{1}+b_{2}\right) \quad \text { and } \quad \frac{z_{2} G^{\prime}\left(z_{2}\right)}{G\left(z_{2}\right)}=\frac{m i}{2}\left(b_{1}+b_{2}\right),
$$

where

$$
m \geq \frac{1-|\varepsilon|}{1+|\varepsilon|} \quad \text { and } \quad \varepsilon=i \tan \frac{\pi}{4}\left(\frac{b_{2}-b_{1}}{b_{1}+b_{2}}\right)
$$

Now, for the case $B \neq-1$, we obtain

$$
\arg \left(\frac{1}{1-\tau}\left(\frac{z_{1}\left(D_{z}^{-\lambda} \mathcal{H}_{\alpha, \beta}^{\gamma+1, k} f\left(z_{1}\right)\right)^{\prime}}{D_{z}^{-\lambda} \mathcal{H}_{\alpha, \beta}^{\gamma+1, k} g\left(z_{1}\right)}-\tau\right)\right)
$$


$=\arg \left(G\left(z_{1}\right)+\frac{z_{1} G^{\prime}\left(z_{1}\right)}{(1-\delta) H\left(z_{1}\right)+\delta+\frac{\gamma-\lambda k}{k}}\right)$

$=\arg \left(G\left(z_{1}\right)\right)+\arg \left(1+\frac{z_{1} G^{\prime}\left(z_{1}\right)}{\left[(1-\delta) H\left(z_{1}\right)+\delta+\frac{\gamma-\lambda k}{k}\right] G\left(z_{1}\right)}\right)$

$=-\frac{\pi}{2} b_{1}+\arg \left(1-\frac{m i}{2 \rho}\left(b_{1}+b_{2}\right) e^{-i \frac{\pi}{2} \phi}\right)$

$=-\frac{\pi}{2} b_{1}+\arg \left(1-\frac{m}{2 \rho}\left(b_{1}+b_{2}\right) \cos \frac{\pi}{2}(1-\phi)+\frac{m i}{2 \rho}\left(b_{1}+b_{2}\right) \sin \frac{\pi}{2}(1-\phi)\right)$

$\leq-\frac{\pi}{2} b_{1}-\tan ^{-1}\left(\frac{m\left(b_{1}+b_{2}\right) \sin \frac{\pi}{2}(1-\phi)}{2 \rho+m\left(b_{1}+b_{2}\right) \cos \frac{\pi}{2}(1-\phi)}\right)$

$\leq-\frac{\pi}{2} b_{1}-\tan ^{-1}\left(\frac{(1-|\varepsilon|)\left(b_{1}+b_{2}\right) \cos \frac{\pi}{2} t}{2(1+|\varepsilon|)\left(\frac{(1+A)(1-\delta)}{1+B}+\delta+\frac{\gamma-\lambda k}{k}\right)+(1-|\varepsilon|)\left(b_{1}+b_{2}\right) \sin \frac{\pi}{2} t}\right)$

$=-\frac{\pi}{2} a_{1}$,

where $a_{1}$ and $t$ are given by (2.8) and (2.10), respectively.

Also,

$$
\begin{aligned}
& \arg \left(\frac{1}{1-\tau}\left(\frac{z_{2}\left(D_{z}^{-\lambda} \mathcal{H}_{\alpha, \beta}^{\gamma+1, k} f\left(z_{2}\right)\right)^{\prime}}{D_{z}^{-\lambda} \mathcal{H}_{\alpha, \beta}^{\gamma+1, k} g\left(z_{2}\right)}-\tau\right)\right) \\
& \geq \frac{\pi}{2} b_{2}+\tan ^{-1}\left(\frac{(1-|\varepsilon|)\left(b_{1}+b_{2}\right) \cos \frac{\pi}{2} t}{2(1+|\varepsilon|)\left(\frac{(1+A)(1-\delta)}{1+B}+\delta+\frac{\gamma-\lambda k}{k}\right)+(1-|\varepsilon|)\left(b_{1}+b_{2}\right) \sin \frac{\pi}{2} t}\right)
\end{aligned}
$$




$$
=\frac{\pi}{2} a_{2}
$$

where $a_{2}$ and $t$ are given by (2.9) and (2.10), respectively.

Similarly, for the case $B=-1$, we have

$$
\arg \left(\frac{1}{1-\tau}\left(\frac{z_{1}\left(D_{z}^{-\lambda} \mathcal{H}_{\alpha, \beta}^{\gamma+1, k} f\left(z_{1}\right)\right)^{\prime}}{D_{z}^{-\lambda} \mathcal{H}_{\alpha, \beta}^{\gamma+1, k} g\left(z_{1}\right)}-\tau\right) \leq-\frac{\pi}{2} b_{1}\right.
$$

and

$$
\arg \left(\frac{1}{1-\tau}\left(\frac{z_{2}\left(D_{z}^{-\lambda} \mathcal{H}_{\alpha, \beta}^{\gamma+1, k} f\left(z_{2}\right)\right)^{\prime}}{D_{z}^{-\lambda} \mathcal{H}_{\alpha, \beta}^{\gamma+1, k} g\left(z_{2}\right)}-\tau\right) \geq \frac{\pi}{2} b_{2}\right.
$$

The above two cases contradict the assumptions. Consequently, the proof of the theorem is complete.

\section{References}

[1] A. A. Attiya, Some applications of Mittag-Leffler function in the unit disk, Filomat 30(7) (2016), 2075-2081.

[2] A. Ebadian, S. Shams, Z. G. Wang and Y. Sun, A class of multivalent analytic functions involving the generalized Jung-Kim-Srivastava operator, Acta Univ. Apulensis 18 (2009), 265-277.

[3] P. J. Eenigenburg, S. S. Miller, P. T. Mocanu and M. O. Reade, On a Briot-Bouquet differential subordination, in: General Inequalities 3, vol. 64 of International Series of Numerical Mathematics, pp. 339-348, Birkhäuser, Basel, 1983.

[4] M. Garg, P. Manoha and S. L. Kalla, A Mittag-Leffler-type function of two variables, Integral Transforms Spec. Funct. 24(11) (2013), 934-944.

[5] V. Kiryakova, The multi-index Mittag-Leffler functions as an important class of special functions of fractional calculus, Comput. Math. Appl. 59(5) (2010), 1885-1895.

[6] S. S. Miller and P. T. Mocanu, Differential subordinations and univalent functions, Michigan Math. J. 28 (1981), 157-171. 
[7] G. M. Mittag-Leffler, Sur la nouvelle fonction $E_{\alpha}(x)$, C. R. Acad. Sci. Paris 137 (1903), 554-558.

[8] G. M. Mittag-Leffler, Sur la représentation analytique d'une fonction monogène (cinquieme note), Acta Math. 29 (1905), 101-181.

[9] J. C. Prajapati, R. K. Jana, R. K. Saxena and A. K. Shukla, Some results on the generalized Mittag-Leffler function operator, J. Inequal. Appl. 2013, 2013:33, 6 pp.

[10] A. K. Shukla and J. C. Prajapati, On a generalization of Mittag-Leffler function and its properties, J. Math. Anal. Appl. 336 (2007), 797-811.

[11] H. Silverman and E. M. Silvia, Subclasses of starlike functions subordinate to convex functions, Canad. J. Math. 37 (1985), 48-61.

[12] H. M. Srivastava and S. Owa (eds.), Current Topics in Analytic Function Theory, Singapore: World Scientific Publishing Company, 1992.

[13] H. M. Srivastava and Z. Tomovski, Fractional calculus with an integral operator containing a generalized Mittag-Leffler function in the kernal, Appl. Math. Comp. 211 (2009), 198-210. 